\title{
Descrição da artéria celíaca em pombos domésticos (Columba livia)
}

\author{
Cibele Geeverghese ${ }^{1}$ \\ Anne Caroline de Oliveira Barbosa ${ }^{1}$ \\ Marcelle dos Santos Lemos ${ }^{1}$ \\ Gabriel Batista de Oliveira Borges ${ }^{1}$ \\ Marcelo Ismar Santana ${ }^{2 *}$ \\ Eduardo Maurício Mendes de Lima ${ }^{2}$ \\ Universidade de Brasília, Faculdade de Agronomia e Medicina Veterinária \\ ${ }^{1}$ Curso de Medicina Veterinária \\ ${ }^{2}$ Laboratório de Anatomia, ICC Ala Sul, Campus Universitário Darcy Ribeiro \\ Caixa Postal 4508, CEP 70.910-970, Brasília - DF, Brasil \\ * Autor para correspondência \\ misantana@unb.br
}

Submetido em 06/09/2011

Aceito para publicação em 10/02/2012

\section{Resumo}

Este estudo objetivou definir a origem e a distribuição da artéria celíaca e de seus ramos colaterais em 15 aves da espécie Columba livia, cedidas pelo Centro de Controle de Zoonoses de Brasília. Com a finalidade de marcar o sistema arterial dos espécimes, o tronco braquiocefálico esquerdo foi canulado e injetado com solução aquosa de látex corado. Posteriormente, procedeu-se à fixação das aves com solução aquosa de formol 10\% v/v e a dissecação com instrumentos adequados, obtendo-se os resultados descritos neste artigo. A artéria celíaca originou-se da face ventral da aorta descendente. O primeiro ramo colateral surgiu da própria artéria celíaca, constituindo a artéria esofágica. Posteriormente, a artéria celíaca se bifurcou em dois ramos, denominados ramo esquerdo e ramo direito da artéria celíaca. O ramo esquerdo emitiu a artéria proventricular ventral, seguida das artérias esplênicas, da artéria proventricular dorsal e da artéria hepática esquerda. O ramo esquerdo se bifurcou, originando as artérias gástricas, ventral e esquerda. O ramo direito emitiu a artéria hepática direita, seguida da artéria ileal e da artéria gástrica direita. Por fim, o ramo direito prosseguiu como artéria pancreaticoduodenal. Os achados deste trabalho apresentaram grande similaridade com as linhagens de aves da espécie Gallus gallus, exceto pela ausência da artéria ileocecal, de ramos císticos e da artéria gástrica dorsal.

Palavras-chave: Artéria celíaca; Columba lívia; Distribuição; Origem; Pombo doméstico

\section{Abstract}

Description of the celiac artery in domestic pigeons (Columba livia). This paper aimed to define the origin and distribution of the celiac artery and its collateral branches in 15 fowls from the Columba livia species, which were obtained from the Zoonosis Control Center of Brasilia, Brazil. In order to mark the arterial 
system of the specimens, the left brachiocephalic trunk was canullated and a colored water-latex solution was injected there. Afterwards, fowls were fixed in a $10 \% \mathrm{v} / \mathrm{v}$ formaldehyde solution and dissected with appropriate equipment, presenting the results described in this paper. The celiac artery originated from the ventral face of the descendent aorta. The first collateral branch arose from the celiac artery itself, forming the esophageal artery. Then, the celiac artery has bifurcated into two branches, named left and right branches of the celiac artery. The left branch emitted the proventricular ventral artery, followed by the splenic arteries, proventricular dorsal artery, and the left hepatic artery. The left branch has bifurcated into two branches, known as ventral and left gastric arteries. The right branch emitted the right hepatic artery, followed by the ileal artery and the right gastric artery. Finally, the right branch turned into the pancreaticoduodenal artery. Our findings showed a great similarity with the avian lineages of the Gallus gallus species, except for the lack of ileocecal artery, cystic branches, and dorsal gastric artery.

Key words: Celiac artery; Columba livia; Distribution; Domestic pigeon; Origin

\section{Introdução}

A família dos Columbidae (ordem Columbiformes) é originária da Eurásia e África, sendo amplamente distribuída pelo planeta por possuir uma capacidade enorme de adaptação a diversos tipos de habitat, tais como savanas, campos, áreas cultivadas ou até mesmo grandes cidades. A espécie Columba livia, em específico, é bastante encontrada em países da América do Sul, tais como Brasil, Bolívia, Chile e Peru (RIBEIRO et al., 1995).

O fenótipo do pombo doméstico é geralmente cinzento claro, com uma mancha púrpura e verde lateralmente ao pescoço, o bico cinzento e os pés laranjaavermelhados (SOUSA, 2004).

A alimentação de pombos adultos campestres constitui-se basicamente de grãos integrais (SALLES et al., 2002), já os que vivem nas cidades, acabam ingerindo restos de alimento encontrados no chão, inclusive resíduos, muitas vezes contaminados (SIGRIST, 2006).

O estudo dos pombos é importante por diversos aspectos: 1) ecológicos, por serem aves onívoras que habitam diversos ambientes, inclusive grandes cidades; 2) sociais, por entupirem calhas, apodrecerem forros de madeira e causarem danos a monumentos históricos e pinturas de carros; 3 ) epidemiológicos, por alojarem parasitas e transmitirem doenças como Toxoplasmose, Criptococose, Ornitose, Histoplasmose e Salmonelose, além de dermatites (RIBEIRO et al., 1995).

Entre os aspectos citados, aqueles relacionados com sua alimentação são os mais relevantes em vista do enfoque deste trabalho, que visa descrever a origem e a distribuição da artéria celíaca em pombos domésticos (Columba livia). A alimentação diversificada dos pombos torna ainda mais interessante esse estudo, por se tratar de um vaso que irriga em grande extensão o aparelho digestório, possibilitando uma melhor compreensão do seu funcionamento.

Dessa forma, o presente estudo objetivou estudar a origem e a distribuição da artéria celíaca e de seus ramos colaterais em pombos domésticos (Columba livia) e compará-la com a de outras espécies de aves domésticas e silvestres.

\section{Materiais e Métodos}

Para a realização deste trabalho, foram utilizados 15 exemplares de Columba livia (J. F. Gmelin, 1789) de ambos os gêneros. As aves foram cedidas pelo Centro de Controle de Zoonoses de Brasília - DF ao Laboratório de Patologia Animal da Faculdade de Agronomia e Medicina Veterinária da Universidade de Brasília, responsável pela avaliação da causa da morte dos animais, sendo posteriormente repassadas ao Laboratório de Anatomia Veterinária da mesma unidade.

Com a finalidade de marcar o sistema arterial dos espécimes, as aves tiveram a cavidade celomática aberta através da secção mediana e longitudinal do osso esterno e da respectiva musculatura peitoral até seu terço médio, o que permitiu o isolamento e a canulação do tronco braquiocefálico esquerdo, para imediata injeção de solução aquosa de látex corado, por meio de pressão manual constante. 
Em seguida, as aves foram fixadas em solução aquosa de formol $10 \% \mathrm{v} / \mathrm{v}$ e acondicionadas em recipientes adequados.

Os exemplares foram dissecados com a utilização de instrumentos cirúrgicos apropriados e, quando necessário, com o auxílio de uma lupa monocular. Concomitantemente, foram realizadas anotações e desenhos esquemáticos sobre a origem, o número e a ordenação da artéria celíaca e seus ramos em cada espécime analisado (Figura 1).

A denominação das artérias teve como base os termos determinados pela Nomina Anatômica Aviária (BAUMEL, 1979).

\section{Resultados}

Nos pombos dissecados, a artéria celíaca originouse da face ventral da aorta descendente, porção abdominal. O primeiro ramo colateral surgiu da própria artéria celíaca, a $3 \mathrm{~mm}$ de sua origem, constituindo a artéria esofágica. Em quatro exemplares $(27 \%)$, foi observada a emissão de duas artérias esofágicas oriundas diretamente da artéria celíaca, e, em 11 exemplares (73\%), somente uma artéria esofágica foi emitida. Após uma média de $9 \mathrm{~mm}$ a partir de sua origem, a artéria celíaca se bifurcou em dois ramos, denominados ramo esquerdo da artéria celíaca e ramo direito da artéria celíaca, este último de maior extensão e calibre.

FIGURA 1: Esquematização da distribuição da artéria celíaca em pombos domésticos (Columba livia). A-Aorta descendente abdominal; B - Artéria celíaca; C - Artéria esofágica; D - Ramo esquerdo da artéria celíaca; E - Ramo direito da artéria celíaca; F Artéria proventricular ventral; G - Artérias esplênicas; H - Artéria proventricular dorsal; I - Artéria hepática esquerda; J - Artéria gástrica esquerda; $\mathrm{K}$ - Artéria gástrica ventral; L - Artéria hepática direita; $\mathrm{M}$ - Artéria gástrica direita; $\mathrm{N}$ Artéria pancreaticoduodenal; O - Ramos para duodeno e pâncreas; P - Artéria ileal; Q - Ramos pilóricos; 1 - Esôfago; 2 Proventrículo; 3 - Ventrículo; 4 - Baço; 5 - Pâncreas; 6 - Duodeno; 7 - Íleo.

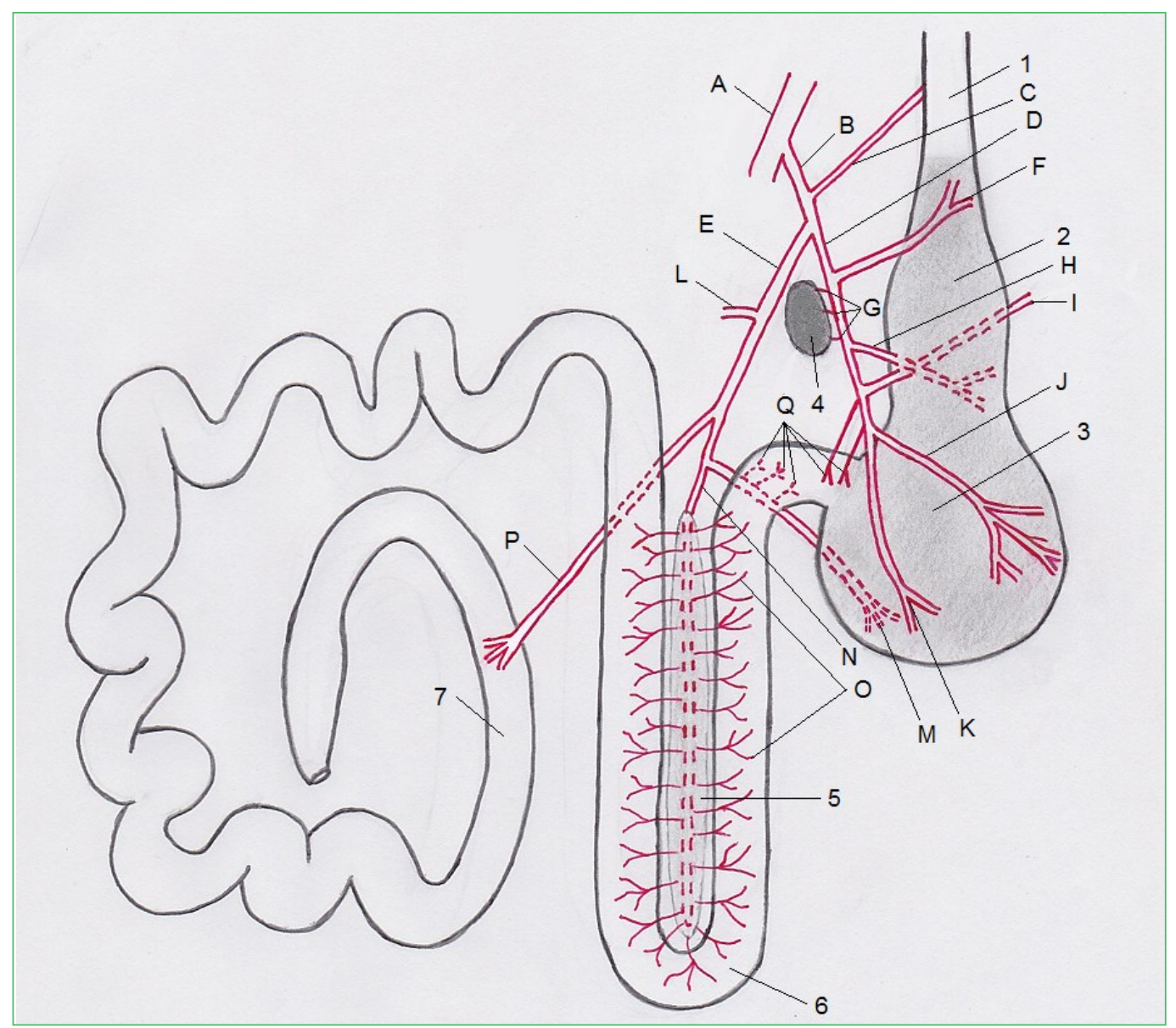


O ramo esquerdo da artéria celíaca emitiu a artéria proventricular ventral $5 \mathrm{~mm}$ após a bifurcação, seguida das artérias esplênicas, da artéria proventricular dorsal e da artéria hepática esquerda. A artéria proventricular ventral seguiu um curso cranial, irrigando principalmente a porção proximal e a face ventral do proventrículo; enquanto a proventricular dorsal seguiu caudalmente, irrigando principalmente a porção distal e a face dorsal do proventrículo. As artérias esplênicas, de diâmetro e extensão reduzidos, variaram em número de dois a quatro, irrigando toda a extensão do baço. A artéria hepática esquerda originou-se pouco antes da artéria gástrica ventral, irrigando o lobo esquerdo do fígado. Por fim, o ramo esquerdo se bifurcou, originando a artéria gástrica ventral, que emitiu de dois a três ramos gástricos, e a artéria gástrica esquerda, que irrigou a face lateral esquerda do ventrículo com seus diversos ramos. Em 13 exemplares (87\%), logo após a bifurcação, o ramo esquerdo emitiu de um a dois ramos pilóricos, e em dois exemplares (13\%), o ramo direito forneceu esses ramos.

O ramo direito da artéria celíaca emitiu a artéria hepática direita $12 \mathrm{~mm}$ após a bifurcação, enviando ramos para o lobo direito do fígado. Em seguida, foi emitida a artéria ileal, que enviou ramos somente para o íleo, e a artéria gástrica direita, que emitiu alguns ramos pilóricos para a porção proximal do duodeno e de três a quatro ramos para a face lateral direita do ventrículo. Por fim, o ramo direito prosseguiu como artéria pancreaticoduodenal, emitindo de 21 a 35 ramos para pâncreas e duodeno. Em 1 exemplar (7\%), excepcionalmente, $\log$ após a bifurcação da artéria celíaca, o ramo direito emitiu uma artéria duodenal destinada à porção proximal do duodeno.

\section{Discussão}

A ramificação da artéria celíaca nos pombos domésticos estudados assemelha-se ao descrito em pombos por Bhaduri et al. (1957), que relataram a distribuição de seus ramos para os seguintes órgãos: proventrículo, ventrículo, fígado, baço, pâncreas e parte do intestino delgado. No entanto, estavam ausentes os cecos e a vesícula biliar em todos os pombos dissecados, característica que também foi observada por Gonçalves et al. (2011) em papagaios verdadeiros (Amazona aestiva) e, que podem estar relacionadas com o caminho evolutivo das espécies, bem como com a alta taxa de passagem dos alimentos pelo canal alimentar.

A artéria esofágica como primeiro ramo colateral emitido diretamente da artéria celíaca está de acordo com os trabalhos realizados em pombos domésticos por Bhaduri et al. (1957), no galo doméstico por Schwarze e Schröder (1972) e Kuru (2010) e no ganso doméstico por Malinovsky e Visnanská (1975), além do que foi descrito para galos e galinhas das linhagens Label Rouge, Dekalb White, Label Rouge T44N e Bovans Goldline (PERISSOTTO et al., 2001; MIRANDA et al., 2006; MIRANDA; SILVA, 2007; PERISSOTTO; SILVA, 2007), enquanto no murucututu (SILVA et al., 2009a), na arara canindé (SILVA et al., 2009b), na suindara (SILVA et al., 2009c) e nas aves das linhagens Ross e Peterson (SILVA et al., 1997; CARDOSO et al., 2000) o primeiro ramo enviado pela artéria celíaca foi a artéria proventricular dorsal. Nos pombos analisados, a artéria proventricular dorsal originou-se do ramo esquerdo da artéria celíaca, após a emissão das artérias esplênicas.

Em concordância com o descrito em patos (PINTO et al., 1998), aves da linhagem Label Rouge (PERISSOTTO et al., 2001) e falcões vermelhos (HALIGUR; DUZLER, 2010), a artéria proventricular ventral constituiu a primeira emissão do ramo esquerdo da artéria celíaca. Diferindo deste padrão, em perus (GADHOKE et al., 1975), ambas artérias proventriculares se originaram diretamente da artéria celíaca, logo após a emissão da artéria esofágica.

A forma de irrigação do ventrículo aproximou-se da observada em patos e em aves das linhagens Label Rouge, Dekalb White, Label Rouge T44N e Bovans Goldline de autores já citados, e também da linhagem Peterson (CARDOSO et al., 2000), Redbro Plumé (MIRANDA et al., 2005) e em mutuns (GONÇALVES et al., 2010), nos quais foi relatada a presença das artérias gástrica ventral e gástrica esquerda, provenientes do ramo esquerdo da artéria celíaca, e da artéria gástrica direita, emitida pelo ramo direito. Porém, diferiu em todos os casos quanto à artéria gástrica dorsal, encontrada em todas as espécies citadas, mas ausente em $100 \%$ dos exemplares estudados e, em relação à 
artéria gastroduodenal, presente na maioria das aves, mas também ausente nos espécimes descritos neste trabalho. Tal irrigação peculiar do ventrículo por apenas três ramos, enquanto na maioria das espécies esse órgão recebe quatro ramos, pode ter ocorrido evolutivamente devido a uma adaptação à alimentação onívora altamente diversificada, e que pode ter como consequência uma diminuição da eficiência digestiva, aumentado a taxa de passagem. O contrário foi relatado em papagaios verdadeiros (GONÇALVES et al., 2011), cujo grande número de ramos enviados ao proventrículo e ventrículo está provavelmente relacionado à grande eficiência alimentar dessas aves.

Assim como observado para diferentes linhagens do gênero Gallus (BHADURI et al., 1957; MALINOVSKY; NOVOTNÁ, 1977; NICKEL et al., 1977; GETTY, 1986), no ganso doméstico (MALINOVSKY; VISNANSKÁ, 1975) e em perus (GADHOKE et al., 1975), o ramo esquerdo da artéria celíaca deu origem à delgada artéria hepática esquerda, enquanto o ramo direito deu origem à artéria hepática direita.

No entanto, como variação de padrão, foi encontrado por Malinovsky e Visnanská (1975), Silva et al. (2009c), Kuru (2010) e Gonçalves et al. (2011), que a artéria hepática esquerda pode ser ramo direto da artéria gástrica ventral, o que pode representar uma característica individual, tanto para o galo doméstico como para outras espécies de aves, e que nada interfere na fisiologia hepática.

Também, na maioria das espécies descritas (RAFAEL et al., 2005; SILVA et al., 2005), é de se esperar que a artéria hepática direita envie pelo menos um ramo cístico para a vesícula biliar. No caso dos pombos domésticos, assim como reportado por Gonçalves et al. (2011) em papagaios verdadeiros, a ausência de vesícula biliar levou à ausência de ramos císticos.

Em aves do gênero Gallus, das linhagens Ross, Peterson e Arbor Acres (SILVA et al., 1997; CARDOSO et al., 2000; RAFAEL et al., 2005), os ramos esplênicos originaram-se somente do ramo direito da artéria celíaca reportada, descrição corroborada pelos autores Schwarze e Schröder (1972), Malinovsky et al. (1973), Baumel (1979), Getty (1986) e Haligur e Duzler (2010). No entanto, nos pombos dissecados, bem como nos relatos de Malinovsky e Visnanská (1975) e nas linhagens Cobb 500, Dekalb White e Bovans Goldline (SILVA et al., 2005; MIRANDA et al., 2006; MIRANDA; SILVA, 2007) os ramos esplênicos partiram exclusivamente do ramo esquerdo da artéria, porém com menor frequência. Tal diferença se deve provavelmente ao fato de nessa espécie o ramo esquerdo da artéria celíaca correr juntamente à borda direita do baço, sendo suficiente a emissão de ramos curtíssimos para esse órgão apenas do ramo esquerdo (MALINOVSKY; NOVOTNÁ, 1977).

Como reportado em skuas e papagaios verdadeiros (SILVA et al., 2009d; GONÇALVES et al., 2011) e em aves das linhagens Peterson, Label Rouge, Cobb 500, Label Rouge T44N e Bovans Goldline (CARDOSO et al., 2000; PERISSOTTO et al., 2001; SILVA et al., 2005; MIRANDA; SILVA, 2007; PERISSOTTO; SILVA, 2007), após a emissão da artéria gástrica direita, o ramo direito prosseguiu como artéria pancreaticoduodenal. O número de ramos (21 a 38) enviados para pâncreas e duodeno a partir dessa artéria nas aves estudadas aproxima-se do encontrado nas linhagens Bovans Goldline (16 a 39 ramos), Label Rouge e Label Rouge T44N (20 a 39 ramos) e Peterson (20 a 34 ramos).

Em todas as linhagens da espécie Gallus gallus supracitadas, em skuas (SILVA et al., 2009d), em mutuns (GONÇALVES et al., 2010) e nos relatos de Kuru (2010), foi descrita a emissão de artérias ileocecais a partir da artéria pancreaticoduodenal. No entanto, devido à ausência de cecos nos pombos domésticos, optou-se pela utilização de artéria ileal (BAUMEL, 1979), sendo observada sua emissão diretamente do ramo direito da artéria celíaca após o envio da artéria hepática direita, da mesma forma como relatado para os papagaios verdadeiros (GONÇALVES et al., 2011).

A partir dos resultados foi possível concluir que a origem e o padrão de distribuição da artéria celíaca na espécie Columba livia apresentou grande similaridade com as aves dos gêneros Gallus gallus, Cairina moschata, Pulsatrix perspicillata, Tyto alba e Ara ararauna. Algumas diferenças foram observadas, no entanto, como ausência da artéria ileocecal e de ramos císticos, devido à inexistência de cecos e de vesícula biliar, além da ausência da artéria gástrica dorsal. A 
menor irrigação gástrica devido à ausência da artéria gástrica dorsal, por sua vez, pode estar relacionada à adaptação dessas aves para alta capacidade de digestão de alimentos variados e não para eficiência alimentar. Portanto, a ausência de tais estruturas pode ser resultado de um processo evolutivo, provavelmente devido à adaptação do aparelho digestório à alimentação onívora de alta diversidade.

\section{Referências}

BAUMEL, J. J. Nomina Anatomica Avium. Academic Press: London, 1979. 779 p.

BHADURI, J. L.; BISWAS, B.; DAS, S. K. The arterial system of the domestic pigeon (Columba livia gmelin). Anatomischer Anzeiger, Erlangen, v. 104, n. 14, p. 1-14, 1957.

CARDOSO, J. R.; DRUMMOND, S. S.; MARTINS, A. K.; SILVA, F. O. C.; SEVERINO, R. S.; MOTA, F. C. D. Aspectos anatômicos da artéria celíaca em Gallus gallus da linhagem Peterson. Arquivo de Ciência Veterinária e Zoologia Unipar, Umuarama, v. 3, n. 2 , p. 151-157, 2000.

GADHOKE, J. S.; LINDSAY, R. T.; DESMOND, R. K. Comparative study of the major arterial branches of the descending aorta, and their supply to the abdominal viscera in the domestic turkey (Meleagris gallopavo), Anatomischer Anzeiger, Jena, v. 138, n. 5, p. 438-443, 1975.

GETTY, R. Anatomia dos animais domésticos. Vol. 1. 5 ed. Guanabara Koogan: Rio de Janeiro, 1986. 2000 p.

GONÇALVES, E. S.; SANTANA, M. I.; LIMA, E. M. M.; SILVA, F. O. C.; SEVERINO, R. S.; DRUMMOND, S. S. Origem e distribuição da artéria celíaca em mutuns dos gêneros Crax e Mitu. ARS Veterinaria, Jaboticabal, v. 26, n. 2, p. 88-94, 2010.

GONÇALVES, E. S.; SANTANA, M. I.; ZANCAN, F. T.; PINTO, A. B. F.; LIMA, E. M. M. Distribuição configurada pela artéria celíaca em papagaios-verdadeiros (Amazona aestiva). Arquivo Brasileiro de Medicina Veterinária e Zootecnia, Belo Horizonte, v. 36, n. 5, p. 1141-1148, 2011.

HALIGUR, A.; DUZLER, A. Course and branch of the celiac artery in the red falcon (Buteo rufinus). Veterinarni Medicina, Brno, v. 55, p. 79-86, 2010.

KURU, N. Macroanatomic investigations on the course and distribution of the celiac artery in domestic fowl (Gallus gallus domesticus). Scientific Research and Essays, Sivas, v. 5, n. 23, p. 3585-3591, 2010.

MALINOVSKY, L.; ROUBAL, P.; VISNANSKA, M. Vascularization of viscera in abdominal part of body cavity in some domestic birds. Folia Morphologica, Prague, v. 21, n. 3, p. 292295, 1973.

MALINOVSKY, L.; VISNANSKA, M. Branching of the celiac artery in some domestic birds, II. The domestic goose. Folia Morphologica, Prague, v. 23, n. 2, p. 128-135, 1975.

MALINOVSKY, L.; NOVOTNA, M. Branching of the coeliac artery in some domestic birds. III. A. Comparison of the pattern of the celiac artery in three breeds of the domestic fowl (Gallus gallus f. domestica). Anatomischer Anzeiger, Jena, v. 141, n. 2, p. 136-146, 1977.

MIRANDA, R. L.; SILVA, F. O. C. Origem e distribuição da artéria celíaca em aves (Gallus gallus) poedeiras de linhagem Bovans Goldline. Revista Horizonte Científico, Uberlândia, v. 1, n. 7, p. 1-15, 2007.

MIRANDA, R. L.; SILVA, F. O. C.; LIMA, J. C. S.; CASTRO, J. R.; QUIRINO, R. C.; SEVERINO, R. S.; DRUMMOND, S. S. Origem e distribuição da artéria celíaca em aves (Gallus gallus) de corte da linhagem Redbro Plumé. Bioscience Journal, Uberlândia, v. 21, n. 3, p. 77-83, 2005

MIRANDA, R. L.; SILVA, F. O. C.; SEVERINO, R. S.; DRUMMOND, S. S.; SOLA, M. C.; MENDONÇA, E. P.; FARIA, A. B. Origem e distribuição da artéria celíaca em aves (Gallus gallus) poedeiras da linhagem Dekalb White. Veterinária Noticias, Uberlândia, v. 12, n. 2, p. 41-46, 2006.

NICKEL, R.; SCHUMMER, A.; SEIFERLE, E. Anatomy of the domestic birds. Berlin: Hamburg: Verlag Paul Parey, 1977. 202 p. PERISSOTTO, D. O.; SILVA, F. O. C. Origem e distribuição da artéria celíaca em aves (Gallus gallus) da linhagem Label Rouge T44 N. Revista Horizonte Cientifico, Uberlândia, v. 1, n. 7, p. 1-15, 2007.

PERISSOTTO, D. O.; SILVA, F. O. C.; SEVERINO, R. S.; DRUMMOND, S. S. Origem e distribuição da artéria celíaca em aves Gallus gallus (matrizes de corte - linhagem Label Rouge). Arquivo de Ciência Veterinária e Zoologia Unipar, Umuarama, v. 4, n. 2, p. 155-161, 2001.

PINTO, M. R. A.; RIBEIRO, A. A. C. M.; SOUZA, W. M. Os arranjos configurados pela artéria celíaca no pato doméstico (Cairina moshata). Brazilian Journal of Veterinary of Research and Animal Science, São Paulo, v. 35, n. 3, p. 103-106, 1998.

RAFAEL, E. L. S.; SILVA, F. O. C.; SEVERINO, R. S.; DRUMMOND, S. S.; BOMBONATO, P. P.; PERES, R. F. G. Origem e distribuição da artéria celíaca em aves (Gallus gallus Linnaeus 1758) da linhagem Arbor Acres. Bioscience Journal, Uberlândia, v. 21, n. 3, p. 55- 60, 2005.

RIBEIRO, M.; TELES, M.; MARUCH, S.; Morphological aspects of the ovary of Columba livia (Gmelin). Revista Brasileira de Zoologia, Curitiba, v. 12, n. 1, p. 151-157, 1995.

SALLES, M. A.; SILVA, P. K. S.; FONSECA, V. R. S.; CARNEIRO, A. L.; BRANCO, F. R.; SILVA, P. L.; ALVES, N. F.; CUNHA, A. P. Pesquisa de Salmonella sp. através de provas de triagem rápida e convencional, em carcaças de frangos abatidos no município de Uberlândia, MG. Higiene Alimentar, Itapetininga, v. 16, n. 92/93, p. 36-40, 2002.

SCHWARZE, E.; SCHRÖDER, L. Compêndio de anatomia veterinária. Vol. 5. Zaragoza: Acribia, 1972. 212 p.

SIGRIST, T. Aves do Brasil: uma visão artística. São Paulo: Avis Brasilis, 2006. 672 p.

SILVA, A. C. J.; BARROS, M. L. G.; NASCIMENTO, H. B.; FREIRE, A. C. B.; PEREIRA, A. P. C.; OLIVEIRA, F. F. Origem e distribuição da artéria celíaca em Murucututu (Pulsatrix perspicillata). In: ENCONTRO INTERNACIONALDEMEDICINA DA CONSERVAÇÃO. MUDANÇAS CLIMÁTICAS, SAÚDE E ECOSSISTEMAS: A SAÚDE NUMA DIMENSÃO ECOLÓGICA, 2, 2009a, Recife. Resumos... Recife: UFRPe, 2009a. p. 119. 
SILVA, A. C. J.; QUEIROZ, R. A.; BANDEIRA, J. T.; SILVEIRA, B. M. R.; CAVALCANTI, L. E. S.; ALBUQUERQUE, M. C. F. Estudo descritivo das artérias celíaca, mesentérica cranial e mesentérica caudal em Arara Canindé (Ara ararauna) In: ENCONTRO INTERNACIONAL DE MEDICINA DA CONSERVAÇÃO. MUDANÇAS CLIMÁTICAS, SAÚDE E ECOSSISTEMAS: A SAÚDE NUMA DIMENSÃO ECOLÓGICA, 2, 2009b, Recife. Resumos... Recife: UFRPe, 2009b. p. 110.

SILVA, A. C. J.; BARROS, M. L. G.; NASCIMENTO, H B.; FREIRE, A. C. B.; PEREIRA, A. P. C.; OlIVEIRA, F. F. Origem e distribuição da artéria celíaca em Suindara (Tyto alba). In: ENCONTRO INTERNACIONAL DE MEDICINA DA CONSERVAÇÃO. MUDANÇAS CLIMÁTICAS, SAÚDE E ECOSSISTEMAS: A SAÚDE NUMA DIMENSÃO ECOLÓGICA, 2, 2009c, Recife. Resumos... Recife: UFRPe, 2009c. p. 119.

SILVA, A. C. J.; BARROS, M. L. G.; NASCIMENTO, H. B.; FREIRE, A. C. B.; PEREIRA, A. P. C.; OLIVEIRA, F. F. Descrição das artérias celíaca, mesentérica cranial e mesentérica caudal em Skua (Stercorarius skua). In: ENCONTRO INTERNACIONAL DE MEDICINA DA CONSERVAÇÃO. MUDANÇAS CLIMÁTICAS, SAÚDE E ECOSSISTEMAS: A SAÚDE NUMA DIMENSÃO ECOLOGICA, 2, 2009d, Recife. Resumos... Recife: UFRPe, 2009d. p. 108.
SILVA, F. O. C.; SEVERINO R. S.; SANTOS A. L. Q.; DRUMMOND, S. S.; BOMBONATO, P. P.; SANTANA, M. I. S.; LOPES, D.; MARÇAL, A. V. Origem e distribuição da ateria celíaca em aves (Gallus gallus domesticus, linhagem Ross). Revista Faculdade de Zootecnia, Veterinária e Agronomia, Uruguaiana, Uruguaiana, v. 4, n. 1, p. 35-41, 1997.

SILVA, F. O.; SEVERINO, R. S.; SANTOS,A. L. Q.; DRUMMOND, S. S.; BOMBONATO, P. P.; CAMPOS, D. B.; CAMPOS, A. B.; LIMA, E. M. M.; BORGES, A. C.; MARCELINO, E. L. Origem, ramificações e distribuições da artéria celíaca em aves fêmeas (Gallus gallus) da linhagem Cobb 500. Bioscience Journal, Uberlândia, v. 21, n. 2, p. 149-154, 2005.

SOUSA, A. J. G. Caracterização ecológica e genética das populações de pombo da rocha (Columba livia) no Parque Natural do Douro Internacional. 2004. Disponível em $<\mathrm{http}$ :/ www.portal.icnb.pt/NR/rdonlyres/49A7B26A-660B-4E8AB160-4C795E3FC1EE/0/PNDIPombodaRocha_Genetica Ecologia 2004.pdf.>. Acesso em: 12 ago. 2011. 